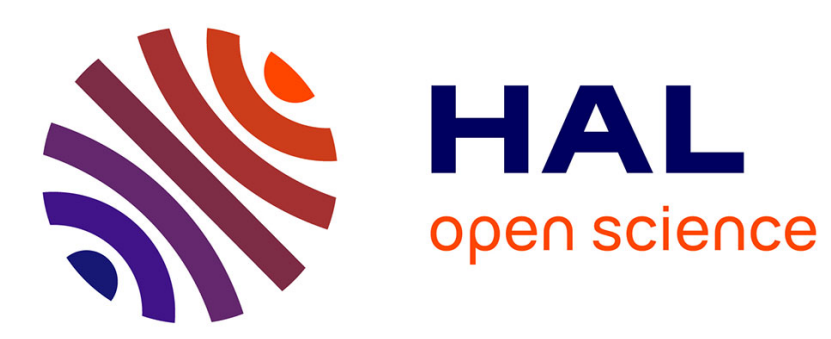

\title{
Data Forwarding Techniques Based on Graph Theory Metrics in Vehicular Social Networks
}

\author{
Anna Maria Vegni, Valeria Loscrì, Pietro Manzoni
}

\section{To cite this version:}

Anna Maria Vegni, Valeria Loscrì, Pietro Manzoni. Data Forwarding Techniques Based on Graph Theory Metrics in Vehicular Social Networks. IEEE PIMRC 2018 - 29th Annual IEEE International Symposium on Personal, Indoor and Mobile Radio Communications, Sep 2018, Bologna, Italy. hal01826237

\section{HAL Id: hal-01826237 https://hal.inria.fr/hal-01826237}

Submitted on 29 Jun 2018

HAL is a multi-disciplinary open access archive for the deposit and dissemination of scientific research documents, whether they are published or not. The documents may come from teaching and research institutions in France or abroad, or from public or private research centers.
L'archive ouverte pluridisciplinaire HAL, est destinée au dépôt et à la diffusion de documents scientifiques de niveau recherche, publiés ou non, émanant des établissements d'enseignement et de recherche français ou étrangers, des laboratoires publics ou privés. 


\title{
Data Forwarding Techniques Based on Graph Theory Metrics in Vehicular Social Networks
}

\author{
Anna Maria Vegni*, Valeria Loscrí ${ }^{\dagger}$, and Pietro Manzoni ${ }^{\ddagger}$ \\ *Dept. of Engineering, Roma Tre University, Rome, Italy. Email: annamaria.vegni@uniroma3.it \\ ${ }^{\dagger}$ FUN Research Team, INRIA Lille-Nord Europe, Lille, France. Email: valeria.loscri@inria.fr \\ ${ }^{\ddagger}$ Computer Engineering Dept., Universitat Politécnica de Valéncia, Valencia, Spain. E-mail: pmanzoni@disca.upv.es
}

\begin{abstract}
Intelligent inter-vehicle communication is a key research field in the context of vehicular networks that applies in real-life applications (e.g., management of accidents, intelligent fuel consumption, smart traffic jams, etc.). Considering different roles of nodes based on their "social aptitude" to relay information could provide a social component in the vehicular structure that can be useful in getting a clear prediction of the topological evolution in time and space proving to be very effective in managing intelligent data forwarding.

In this work we characterize a vehicular network as a graph using the link layer connectivity level and we classify nodes on the basis of specific attributes characterizing their "social aptitude" to forward data. Two forwarding approaches are presented, based on different socialites that allow to $(i)$ select the most social node (i.e., a social hub) or (ii) choose among various social nodes.
\end{abstract}

\section{INTRODUCTION}

Research on vehicular networks is a challenging area due to the rapid topological changes and the high variability of the data links. Nevertheless, several automobile manufactures are investing on research on smart intervehicle communication (IVC) approaches, and a further push towards such direction is also coming by the advent of Internet of Vehicles (IoV) where different kinds of interactions among vehicles (V2V), vehicle and infrastructures (V2I) or vehicle and pedestrian (V2P) can be envisaged [1]. On a parallel (research) lane, wireless social networks have gained their place in the context of the Internet of Things (IoT) paradigm by creating straightforward social interactions among objects [2].

By considering that individuals also play a central role in vehicular networks, the concept of Vehicular Social Networks (VSNs) has appeared [3], [4], considered as a special class of VANETs based on the integration of social aspects and features. On the other hand, the topological evolution of vehicular networks has been investigated in several works (e.g., [5], [6]), exploiting structural analysis and statistical measures for graph analysis. A key challenge to design efficient data forwarding approaches in vehicular environments based on graphs properties is the knowledge and update of the topological characteristics of the underlying VSNs, that can be achieved through service-oriented architecture and cloud computing [7].

For this purpose, in this paper we present a fogbased architecture based on the results obtained in [8], where information can assist in the acquisition of the necessary information to gather specific metrics such as central betweenness, local clustering, node degree, etc. Information about such graph metrics can be then exploited to analyze the social behavior of the network nodes. Knowing how social is a node allows to select a next-hop forwarder that is able to well disseminate a message. Indeed, if a node is "socially active" it is expected to be willing to retransmit a message within the network, while a no social node does not represent an optimal choice for data retransmission. Determining how social a node is, represents an important challenge. Indeed, if a node is elected as "too social", then we can encounter multiple copies of a message then affecting the network load; on the other side, a node assumed as "poor social" will not be selected as next hop forwarder, thus providing further delay in the transmission phase.

In this paper, we consider two main contributions:

- We present a fog-based architecture in order to represent a real-world based solution to gather the needed information for the forwarding approaches proposed;

- We integrate graph properties and metrics in the forwarding decision design and we analyze the different metrics in two representative vehicular network scenarios;

In particular, we evaluate two message forwarding techniques based one on the betweenness metric, called "GrAph MEtrics based forwarding technique (GAME)", and another one on the betweenness and local clustering coefficient, called "Small-WORID based ForwardIng technique for Social Hub (SWORDFISH)". By analyzing the vehicular social degrees derived from such techniques, we can infer interesting features of both the approaches. Betweenness and local clustering are associated with a kind of "willingness" of a node to play the relay role. In practice, higher values of betweenness of nodes in a network are associated with a higher aptitude 
to forward information to other nodes. Following the simulation results in this paper, we can conclude that GAME seems to be more "node-selective" by choosing very few nodes (i.e., hubs) with high social activities, while SWORDFISH allows the selection of a pool of nodes to be eligible as forwarders.

The rest of the paper is organized as follows. Section II describes a fog-based architecture that allows to gather necessary information about graph theory metrics associated to the vehicular network. Such information is taken into account in order to compute the vehicular social degrees. In Section III we present two message forwarding techniques (i.e., GAME and SWORDFISH) and the analysis of their graph-based features in two different traffic scenarios (i.e., sparse and dense traffic scenarios). In Section IV we describe the behavior of GAME and SWORDFISH in terms of forwarding probability. Finally, conclusions are drawn at the end of this paper.

\section{Fog-BASED ARChitecture}

The concept of "node" representing a vehicle is here extended using the idea of fog computing. Following the results in [8], in this paper we consider each vehicle as a "content island" capable of interconnecting groups of "things" packed-up together to interchange data and processing among themselves and with other content islands. A content island is a publish/subscribe based system where a set of "things", capable of some type of processing and sensing, interchange messages inside the island and with other islands, talking MQTT. The island core allows to directly handle the flow of messages inside the island and among the islands.

In this context a message can represent: $(i)$ a collection of data, (ii) a processing or data analysis request, or (iii) an action trigger, and can be interchanged among nodes that either belong to the same island or to other islands. A collection of data is clearly a set of values obtained from a source inside an island, like the evolution of the battery charge in an electric vehicle during a day. A processing or data analysis request can indicate the need for data intensive processing over a data-set, like for example computing the linear relationship between a response (humidity) and one or more predictive terms (temperature). Finally, an action trigger can be a request to, if certain conditions hold, to turn off a device, or publish a message to Twitter. Three elements i.e., (i) the MQTT broker, (ii) the DTN daemon, and (iii) the MQTT near-user edge gateway (MQTT-NEG), constitute the island core. These elements will typically be executing in the same specific node of the island.

Two classes of messages are used: local messages and global messages. Global messages are both distributed inside the island and forwarded to other islands by the DTN daemon. The basic connectivity inside the island

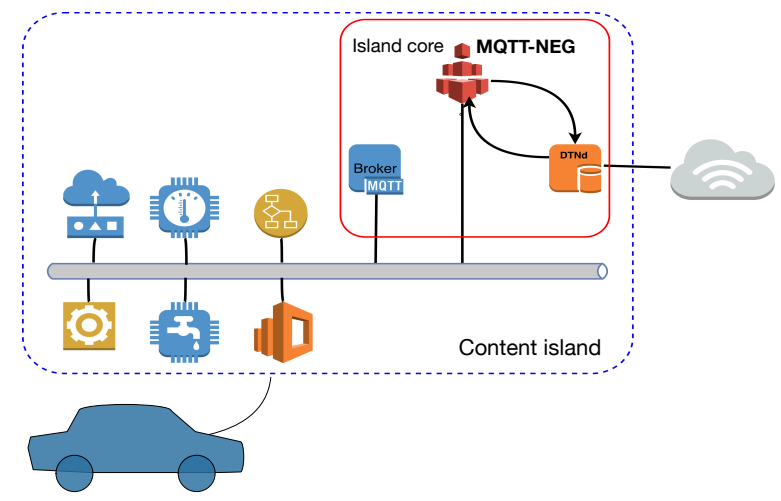

Fig. 1. Content island overall architecture as part of a vehicle.

can take place using any common standard technology, like Ethernet, Wi-Fi, etc.

Inter-islands connectivity is where the VSN and the forwarding techniques described in the following sections come into play, giving the possibility to distribute information, create "conversations" among the members of specific groups of islands, and deploy applications allowing users to cooperate.

\section{PRoposed APPROACHES}

Mobile social networks make use of the often periodic physical locality patterns of the users to improve their knowledge about opportunities to interact and take advantage of the proximity among nodes to form spontaneous temporal communities.

Nodes localization can be performed using dedicated low-cost HW capable of tracking information about other close-by devices, sensing their physical layer activities, e.g., BT beaconing, WiFi SSID beaconing and correlate these information with GPS coordinates. All these data can be used to accumulate knowledge of the neighborhood status and how it evolves and how it organizes in time, according to short term patterns (i.e., minutes, hours) to longer term patterns (i.e., days or weeks). Moreover, nodes can periodically broadcast profiling data related with the topics they are interested in, using a predefined format.

In this paper, we describe two multi-hop forwarding probabilistic techniques, based on some graph theory metrics, namely: distance, betweenness and clustering coefficient. The forwarding decision mechanism occurs when a source vehicle needs to re-transmit a message within its transmission range. Potential forwarders distinguish based on vehicular social degree, defined according to specific graph metric. Higher the vehicular social degree is, higher the vehicle's forwarding probability is expected.

The first approach, called GAME ("GrAph MEtrics based forwarding technique") exploits the information about node betweenness. Let us assume that for the $i$-th 
node in the network it is available the information about its betweenness i.e., $B_{i}$, since a fog-based architecture as explained in Section II is considered. The probability that the $i$-th node is defined as "social active" i.e., $P_{s, i}^{G A M E}$, since it shows a high value of betweenness $B_{i}$, is given as:

$$
P_{s, i}^{G A M E}=\log \left(1+\frac{B_{i}}{\max (\mathbf{B})}\right)
$$

where $\mathbf{B}$ is the betweenness vector of all the $N$ vehicles in the network, i.e., $\mathbf{B}=\left[B_{1}, B_{2}, \ldots, B_{N}\right]$. Mathematically, the betweenness represents the measure of centrality of a node in a graph $G=(E, V)$, based on shortest paths i.e.,

$$
B_{i}=\sum_{\substack{s \neq i \neq t \\ s, i, t \in V}} \frac{\rho_{s t}(i)}{\rho_{s t}}
$$

where $\rho_{s t}$ is the number of shortest paths from vehicle $s$ to $t$, and $\rho_{s t}(i)$ is the number of shortest paths from $s$ to $t$ passing through node $i$. Notice that $B_{i}$ is a measure of the global importance of vehicle $i$; as a consequence, a vehicle with high betweenness centrality plays a crucial role in the connectivity of the network, since the higher $B_{i}$, more the number of shortest paths in the network passing through the vehicle $i$. In our scenario, for every pair of vehicles, there exists at least one shortest path between them such that the number of hops that the path passes through is minimized. Moreover, if a node is more central, then it is expected to be able to forward a message to a larger number of neighbors.

However, information about betweenness only can be limitative in order to detect a social vehicle, specially if a node is not so central but its neighbors are central. Another important metric that needs to be considered is the local clustering coefficient (LCC), which captures the degree to which the neighbors of a given node link to each other i.e.,

$$
L C C_{i}=\frac{2 L_{i}}{k_{i}\left(k_{i}-1\right)},
$$

where $L_{i}$ represents the number of links between the $k_{i}$ neighbors of the $i$-th node. The local clustering coefficient gives information if two neighbors of a node link to each other, and it is one of the most important metrics for the social network analysis.

Taking into account both betweenness and local clustering allows detecting those social vehicles whose neighbors are expected to be social as well.

In the second technique, SWORDFISH ("SmallWORID based ForwardIng technique for Social Hub"), considers both the betweenness and the local cluster coefficient for each node in the vehicular network graph.
The proposed approach computes the social degree parameter for the $i$-th node, defined as:

$$
\begin{aligned}
P_{s, i}^{S W O R D F I S H} & =\log [1+\lambda]= \\
& =\log \left[1+\frac{L C C_{i} \cdot B_{i}}{L C C_{i}+B_{i}}\right],
\end{aligned}
$$

where $\lambda$ is a parameter that considers both $L C C_{i}$ and $B_{i}$, which are the local clustering coefficient and the betweenness of the $i$-th vehicle, respectively.

According to GAME approach, for low betweenness the vehicular social degree is low, while it increases for higher values of betweenness. Moreover, as expected, high values of social degree are only for a limited number of vehicles. This aspect allows the GAME approach to distinguish social vehicles, which are those with higher values of social degree.

In SWORDFISH the vehicular social degree tends to higher values for higher $\lambda$, while only poor nodes present lower social degree. Indeed, since SWORDFISH vehicular social degree is based not only on betweenness, but also on local clustering coefficient, when the network size increases the number of vehicles elected as social ones is higher. On the other side, for a reduced network size, SWORDFISH will distinguish vehicles with a higher social degree for a fixed $\lambda$. This behavior allows SWORDFISH to select more social vehicles for an increasing number of nodes in the network. Again, this is due since the SWORDFISH social degree considers both $L C C$ and $B$; therefore, if a node has low betweenness but high local clustering coefficient, it is expected to still being a social node. The $L C C$ parameter contains information about the small-world structure of the network graph. Indeed, as known, a small-world structure occurs generally when nodes tend forming clusters and the average distance is low, as well as when nodes have low betweenness and higher centrality.

In Fig. 2 we show the SWORDFISH vehicular social degree versus $\lambda$ for different network sizes. On the other side, in Fig. 3 we depict the GAME vehicular social degree versus the betweenness for different network sizes. We observe that GAME tends to select a poor number of social vehicles, specifically it selects only hub nodes with higher betweenness. Indeed, for higher network size, there is expected a limited number of nodes with high betweenness, while the majority will be with a low betweenness. In general, for low betweenness the social degree is low, while it increases for higher values of betweenness. Moreover, as expected, high values of social degree are only for a limited number of vehicles. This aspect allows the GAME approach to distinguish social vehicles, which are those with higher values of social degree.

Finally, the GAME/SWORDFISH vehicular social degrees are used in the expression of the forwarding 


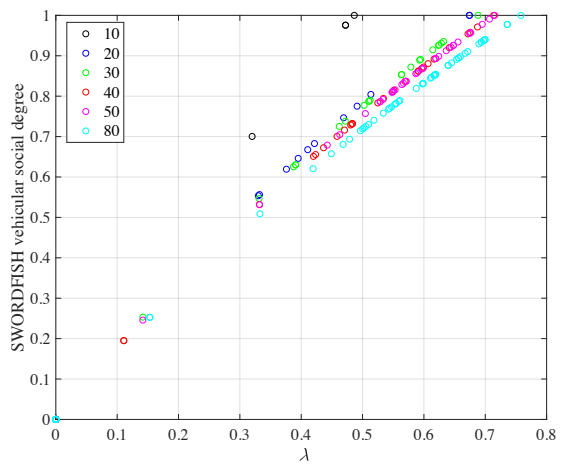

Fig. 2. Normalized values of SWORDFISH vehicular social degree versus $\lambda$ for different network sizes.

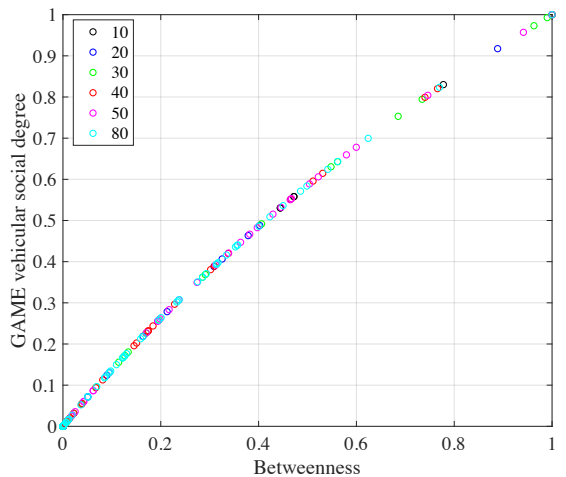

Fig. 3. GAME vehicular social degree versus betweenness for different networks sizes.

probability of the $i$-th vehicle, respectively as follows:

$$
\begin{gathered}
P_{f, i}^{G A M E}=\exp \left[-\rho \frac{z-d_{i}}{P_{s, i}^{G A M E}}\right], \\
P_{f, i}^{S W O R D F I S H}=\exp \left[-\rho \frac{z-d_{i}}{\left.P_{s, i}^{S W O R D F I S H}\right],}\right.
\end{gathered}
$$

where $d_{i}[\mathrm{~m}]$ is the inter-vehicle distance lower than the source transmission range $z[\mathrm{~m}]$ (i.e., $d_{i} \leq z[\mathrm{~m}]$ ), and $\rho$ is the vehicular density $[\mathrm{veh} / \mathrm{m}]$.

\section{Performance Evaluation}

Let us now consider two different scenarios representing a sparse and a dense traffic scenario, comprised of 21 and 41 nodes, respectively. In the first scenario, node ID 21 is a source vehicle that needs to re-transmit a message to one of its neighboring nodes in its transmission range. Similarly, node ID 41 is the source node in the dense traffic scenario. Fig. 4 shows the network graphs that represent the two different network scenarios, respectively.

The network distance computed for each node in the graph with respect to the source vehicle (i.e., node 21 for the sparse traffic scenario and node 41 for the dense traffic scenario) is depicted in Fig. 5 (a) and (b),

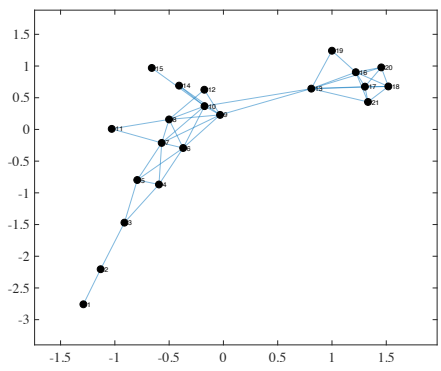

(a)

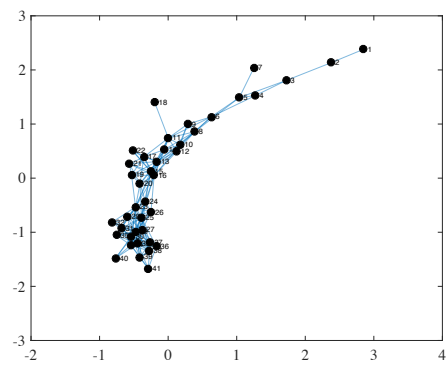

(b)

Fig. 4. Network graphs with (a) 21 nodes (sparse traffic scenario), and (b) 41 nodes (dense traffic scenario).

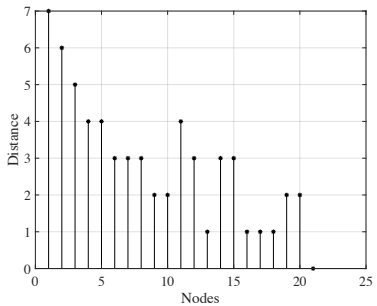

(a)

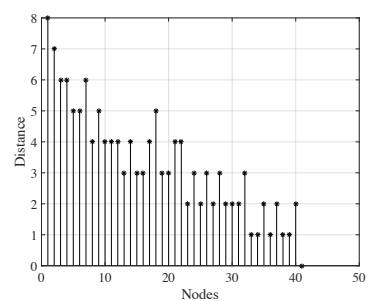

(b)
Fig. 5. Network distance of each node with respect to a source vehicle, specifically (a) node 21 , and (b) node 41.

respectively. For both two scenarios, we also compute the betweenness metric for each node in the networks (see Fig. 6).

For the sparse traffic scenario, the potential next-hop forwarders are only the nodes at 1-hop distance from the source. Therefore, from Fig. 5 (a) the potential eligible forwarders are only nodes 13, 16, 17 and 18.

The forwarding probabilities of the potential next-hop nodes in case of GAME and SWORDFISH approaches are reported in Fig. 7 (a) in case of a sparse traffic scenario with 21 nodes. Here we show the dependence of the forwarding probability for varying distances within the transmission range. As expected for increasing distances the probability will increase as well. Fig. 7 compares GAME to SWORDFISH approach, for different values of 1-hop neighbor social degree $P_{s}$. We observe that GAME has detected one neighbor with highest social degree (i.e., $P_{s}=1$ ), while other curves 


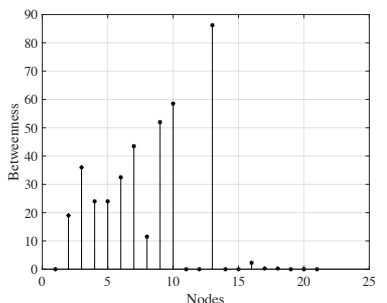

(a)

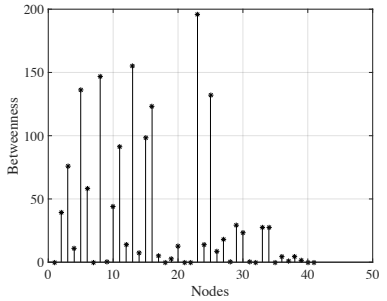

(b)
Fig. 6. Betweenness metric of each node in the network graph with (a) 21 nodes, and (b) 41 nodes.

are for very low vehicular social degrees (i.e., $P_{s}=$ $[0.037,0.0041])$ associated to other 1-hop nodes. On the other side, SWORDFISH associates different values of vehicular social degree (i.e., $P_{s}=[0.66,0.82,0.35]$ ) for each potential 1-hop neighbor. This allows concluding that SWORDFISH is able to select more forwarders with acceptable social degree, while GAME detects that neighbor with highest social degree. SWORDFISH can be well suitable when we need to choose among several social vehicles, while GAME presents its applicability when we need to select the highest social neighbor.

Again, these insights are confirmed when we analyze the forwarding probabilities of the two approaches for the higher node density scenario (i.e., dense traffic scenario), as depicted in Fig. 7 (b). We observe that GAME detects one vehicle with a high social degree, while the other neighbors have lower degree. Consequently, the vehicles with very low social degree will present a very low forwarding probability, and will be likely not selected as next-hop forwarders. On the other side, SWORDFISH has detected neighbors with higher social degree. Likely, for SWORDFISH all these vehicles can be eligible as next-hop forwarders.

\section{CONCLUSIONS}

In this paper we presented two next-hop forwarding techniques based on vehicle social features, taking into account graph theory metric. Betweenness and local clustering coefficient are two main features that highlight how important (i.e., social in the context of social networks) a node is. More social a node is, more effective the data dissemination with the network will be. GAME approach considers the betweenness of a node, so that the node more central in a transmission range will be selected as next-hop forwarder. On the other side, SWORDFISH considers not only the betweenness but also the local clustering coefficient. In this way, SWORDFISH will detect not only central vehicles in a transmission range, but also nodes whose neighbors are social. When traffic data becomes high and the node with the highest sociality is already managing a lot of data to be forwarded, it is better to have an approach

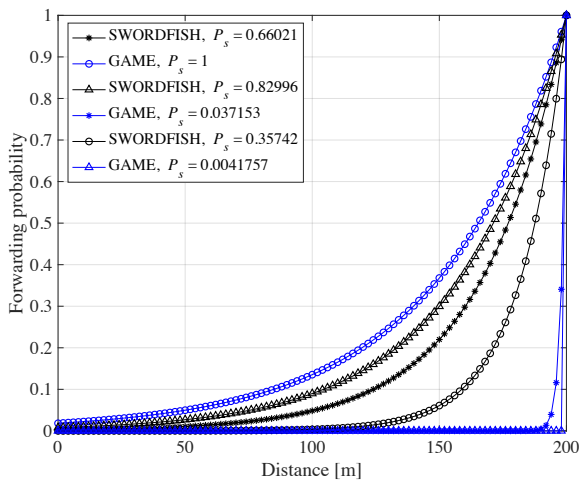

(a)

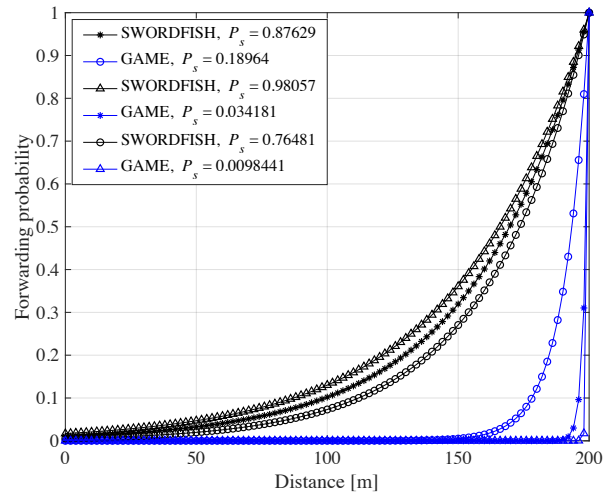

(b)

Fig. 7. GAME/SWORDFISH forwarding probabilities versus distance, for different social degree values associated to 1-hop neighbors of a source vehicle in (a) sparse traffic scenario, and (b) dense traffic scenario.

as SWORDFISH allowing the selections of more social nodes and then more options.

\section{REFERENCES}

[1] L. A. Maglaras, A. H. Al-Bayatti, Y. He, I. Wagner, and H. Janicke, "Social internet of vehicles for smart cities," Journal of Sensor and Actuator Networks, vol. 5, no. 1, 2016.

[2] L. Atzori, A. Iera, G. Morabito, and M. Nitti, "The social internet of things (siot) - when social networks meet the internet of things: Concept, architecture and network characterization," Comput. Netw., vol. 56, no. 16, pp. 3594-3608, Nov. 2012.

[3] A. M. Vegni and V. Loscrí, "A survey on vehicular social networks," IEEE Comm. Surveys \& Tutorials, vol. 17, no. 4, pp. 2397-2419, Fourthquarter 2015.

[4] K. M. Alam, M. Saini, and A. E. Saddik, "Toward social internet of vehicles: Concept, architecture, and applications," IEEE Access, vol. 3, pp. 343-357, 2015

[5] G. Pallis, D. Katsaros, M. D. Dikaiakos, N. Loulloudes, and L. Tassiulas, "On the structure and evolution of vehicular networks," in 2009 IEEE Intl. Symp. on Modeling, Analysis Simulation of Computer and Telecommunication Systems, Sept 2009, pp. 1-10.

[6] M. Fiore and J. Härri, "The networking shape of vehicular mobility," in Proceedings of the 9th ACM IMobiHoc '08. New York, NY, USA: ACM, 2008, pp. 261-272.

[7] K. Alam, M. Saini, and A. E. Saddik, "Workload model based dynamic adaptation of social internet of vehicles," Sensors, vol. 15, no. 9, September 2015.

[8] P. Manzoni, E. Hernández-Orallo, C. T. Calafate, and J.-C. Cano, "A proposal for a publish/subscribe, disruption tolerant content island for fog computing," in Proc. of the 3rd Workshop SMARTOBJECTS. ACM, 2017, pp. 47-52. 